\title{
Coping with Wind Power Uncertainty in Unit Commitment: a Robust Approach using the New Hybrid Metaheuristic DEEPSO
}

\author{
Rui Pinto ${ }^{1,2}$, Leonel M. Carvalho ${ }^{1}$, Jean Sumaili ${ }^{1}$, Mauro S. S. Pinto ${ }^{1}$ and Vladimiro Miranda ${ }^{1,2}$ \\ ${ }^{1}$ INESC TEC and ${ }^{2}$ University of Porto, Portugal \\ rbpinto@inesctec.pt
}

\begin{abstract}
The uncertainty associated with the increasingly wind power penetration in power systems must be considered when performing the traditional day-ahead scheduling of conventional thermal units. This uncertainty can be represented through a set of representative wind power scenarios that take into account the time-dependency between forecasting errors. To create robust Unit Commitment (UC) schedules, it is widely seen that all possible wind power scenarios must be used. However, using all realizations of wind power might be a poor approach and important savings in computational effort can be achieved if only the most representative subset is used. In this paper, the new hybrid metaheuristic DEEPSO and clustering techniques are used in the traditional stochastic formulation of the UC problem to investigate the robustness of the UC schedules with increasing number of wind power scenarios. For this purpose, expected values for operational costs, wind spill, and load curtailment for the UC solutions are compared for a didactic 10 generator test system. The obtained results shown that it is possible to reduce the computation burden of the stochastic UC by using a small set of representative wind power scenarios previously selected from a high number of scenarios covering the entire probability distribution function of the forecasting uncertainty.
\end{abstract}

Index Terms--Unit Commitment, Wind Power Uncertainty, Clustering Techniques, Metaheuristic Optimization.

\section{INTRODUCTION}

The increasing share of wind power in thermal-dominated generating systems is widely seen as a concern not only to the continuity of supply but also to its cost-effective operation. As a matter of fact, the operation of the system can become prohibitively costly and/or intolerably unreliable if wind power forecasts are very different from its actual realization. For example, if the wind power realization is less than the forecast, the system operator will need to start back-up thermal units at increased generating costs and risk load curtailments if the additional capacity is not enough to supply the excess load.

The decision to start or shut down thermal units for the next operating hours must take into account their current

This work is financed by the FCT - Fundação para a Ciência e a Tecnologia (Portuguese Foundation for Science and Technology) within project UID/EEA/50014/2013. operation status and the inherent uncertainty of wind power forecast. Even if wind power could be accurately predicted, there might be hours where wind power is not used at its maximum output due to insufficient ramping capabilities of the generating units. Therefore, robust Unit Commitment procedures are necessary not only to keep operation costs at reasonable levels but also to avoid extreme control actions such as wind spilling and/or load curtailment.

Recent research has been focusing on the development of stochastic methods to solve the UC problem. Wu et al. [1] presented a model to compute the cost of power system reliability based on stochastic optimization of long-term security-constrained UC. Zhao et al. [2] proposed a unified stochastic and robust UC model that takes advantage of both stochastic and robust optimization approaches relying on Benders' decomposition to solve the model efficiently. Xiong et al. [3] proposed a new formulation for a stochastic UC problem that incorporates uncertainty related to the unavailability of generators and load uncertainty. Finally, Zhao et al. [4] proposed an expected value and chance constrained stochastic optimization approach for the UC problem with uncertain wind power output.

The focus of this paper is to study the impact on the total operation costs of using a stochastic approach instead of the traditional deterministic approach. Basically, the stochastic approach consists of using several wind power scenarios to obtain a UC schedule, i.e. the state of the generating units for a given period of time, whereas the deterministic relies solely on the point forecast. The UC model proposed aims at minimizing the expected operational costs over a 24-hour period taking into account key technical constraints of generating units, such as minimum up and down times and ramping rates. Wind power spilling is also considered as well as load curtailment. The wind power scenarios used are obtained from the point forecast taking into account the temporal dependency between forecasting errors [5]. Furthermore, clustering techniques [6] are used to find the set of the most representative scenarios and underlying probabilities. The generation of UC solutions is guaranteed by the new hybrid metaheuristic DEEPSO [7], which is combination of the DE-EP-PSO algorithms, where DE stands for Differential Evolution, EP for Evolutionary Programming and PSO for Particle Swarm Optimization. 
The generation of candidate UC solutions by the metaheuristic DEEPSO contains a simple correction algorithm to make sure that the generating unit constraints, like minimum up and minimum down time, as well as minimum spinning reserve are enforced at all times. The evaluation of solutions consists on the computation of the optimal dynamic economic dispatch for all operating periods taking into account the ramping capabilities of the generating units. The Linear Programming [8] module of the software Gurobi Optimizer $^{\circledR}[9]$ is used for computing the optimal dynamic economic dispatch.

To illustrate the advantages of the methodology proposed, a didactic 10 generator test system [10] is used. This system has been extensively used in other unit commitment research studies. Deterministic and stochastic UC schedules are obtained and the respective expected operation costs over all wind power scenarios compared to make a probabilistic analysis on the wind power spilling and load curtailment risks.

\section{METHODOLOGY}

\section{A. Unit Commitment Model}

The decision variables of the UC problem addressed in this paper are:

$u_{i k}$ - the commitment status of each conventional generating unit $k$ at each period of time $i$, being the value " 1 " the representation of the ON status and the value " 0 " the representation of the OFF status;

$P_{i k}$ - the active power produced by each conventional unit $\mathrm{k}$ at each period of time $i$.

The objective function of the deterministic UC problem is defined as

$$
\begin{gathered}
\min \sum_{i=1}^{T} \sum_{k=1}^{M} u_{i k} \times P C_{k}\left(P_{i k}\right)+u_{i k} \times\left(1-u_{i-1 k}\right) \times S U C_{k} \\
+L L_{i} \times M_{1}+W S_{i} \times M_{2}
\end{gathered}
$$

where $S U C_{k}$ and $P C_{\mathrm{k}}$ are, respectively, the start-up cost and the production cost of generating unit $k, L L_{i}$ and $W S_{i}$ are the load curtailment and the wind spilled of period $i$, respectively, and $M_{1}$ and $M_{2}$ are constants that have a large value, so that load curtailment and wind spill are only activated as a last resort. In this paper, the production cost of each generating unit consists of a 3 straight-line segments approximation [11] of the real quadratic curve.

The constraints of this problem are now enumerated. The first constraint corresponds to the system power balance equation

$$
\sum_{k=1}^{M} u_{i k} \times P_{i k}=\left(P L_{i}-P W_{i}\right)-\left(L L_{i}-W S_{i}\right) \quad \forall_{i}
$$

where $P L_{i}$ and $P W_{i}$ are, respectively, the load and the wind power point forecast for the time period $i$.

The generating units' technical limits are modeled by

$$
\sum_{k=1}^{M} u_{i k} \times \overline{P_{k}} \geq\left(P L_{i}-P W_{i}+P R_{i}\right)-\left(L L_{i}-W S_{i}\right) \quad \forall_{i}
$$

$$
\sum_{k=1}^{M} u_{i k} \times \underline{P_{k}} \leq\left(P L_{i}-P W_{i}\right)-\left(L L_{i}-W S_{i}\right) \quad \forall_{i}
$$

where $P R_{i}$ stands for the spinning reserve of time period $i$. The following constraint represents the active power production boundaries which are related to the physical limits of the generating units.

$$
u_{i k} \times \underline{P_{k}} \leq P_{i k} \leq u_{i k} \times \overline{P_{k}} \quad \forall_{i}, \forall_{k}
$$

The system capability to accommodate wind power depends on the ramping capabilities of the generating units. The variation of the units' power output is limited by the ramp up and ramp down rates, $R U P$ and $R D N$, respectively, as

$$
\begin{array}{ll}
P_{i k}-P_{i-1 k} \leq \Delta t \times R U P_{k} & \forall_{k} \\
P_{i-1 k}-P_{i k} \leq \Delta t \times R D N_{k} & \forall_{k}
\end{array}
$$

where $\Delta t$ is the duration of each individual operating period during the planning schedule, which, in this paper, is equal to 1 hour. The last constraints refer to the minimum up and minimum down time of the generating units. Since $\Delta t=1$, then

$$
\begin{array}{cc}
\left(X O N_{i-1 k}-T U P_{k}\right) \times\left(u_{i-1 k}-u_{i k}\right) \geq 0 & \forall_{i}, \forall_{k} \\
\left(X O F F_{i-1 k}-T D N_{k}\right) \times\left(u_{i k}-u_{i-1 k}\right) \geq 0 & \forall_{i}, \forall_{k}
\end{array}
$$

where $X O N_{i-1 k}$ and $X O F F_{i-1 k}$ are the number of consecutive periods that unit $k$ has been in the $\mathrm{ON}$ and OFF status until period $i-1$, respectively; $T U P_{k}$ and $T D N_{k}$ are the minimum up and down time of unit $k$, respectively. Note that when the unit changes the status, $X O N$ and $X O F F$ must be zeroed.

The stochastic formulation is similar to the deterministic one: the only difference lies in (2), where, instead of minimizing the cost of only one operation scenario, the probability of each scenario is used to compute an expected cost, which is also minimized.

\section{B. DEEPSO}

The new hybrid DEEPSO [3] metaheuristic is a variant of the EPSO (Evolutionary Particle Swarm Optimization). It can either be interpreted as a PSO with self-adaptive properties or an EP method with a self-adaptive recombination operator. DEEPSO won in 2014 a competition organized by the IEEE PES for meta-heuristics in solving the OPF problem.

The general DEEPSO procedure [3] is equal to EPSO. The difference between the two metaheuristics lies in equation used to create new individuals: the Movement Rule. In EPSO, the equations used to create a new individual, $\mathbf{X}_{t}$, from its ancestor, $\mathbf{X}_{t-1}$, from its best ancestor $\mathbf{X}_{b}$, the best ancestor ever found by the population, $\mathbf{X}_{g b}$, and its current velocity $\mathbf{V}_{t}$, are

$$
\begin{gathered}
\mathbf{V}_{t}=w_{i}^{*} \times \mathbf{V}_{t-1}+w_{m}^{*} \times\left(\mathbf{X}_{b}-\mathbf{X}_{t-1}\right)+w_{m}^{*} \times \mathbf{C} \times\left(\mathbf{X}_{g b}^{*}-\mathbf{X}_{t-1}\right) \\
\mathbf{X}_{t}=\mathbf{X}_{t-1}+\mathbf{V}_{t}
\end{gathered}
$$

where $t$ denotes the current generation, the superscript $*$ indicates that the corresponding parameter undergoes evolution under a mutation process, and $\mathbf{C}$ is a $\mathrm{n} \times \mathrm{n}$ diagonal matrix of random variables that follow a Bernoulli distribution with success probability $P$. Matrix $\mathbf{C}$ is randomly sampled every iteration. Typically, the mutation of a generic weight $w$ of an EPSO individual follows a simple additive rule as 


$$
w^{*}=w+\tau N(0,1)
$$

where $\tau$ is the mutation rate, which must be set by the user, and $\mathrm{N}(0,1)$ is a number sampled from the standard Gaussian distribution. Note that the mutated weight must not become negative or greater than 1 .

The DEEPSO movement rule is based on the replacement of the memory term in (10) by the perception term as

$$
\mathbf{V}_{t}=w_{i}^{*} \times \mathbf{V}_{t-1}+w_{m}^{*} \times\left(\mathbf{X}_{r}-\mathbf{X}_{t-1}\right)+w_{m}^{*} \times \mathbf{C} \times\left(\mathbf{X}_{g b}^{*}-\mathbf{X}_{t-1}\right)
$$

where $\mathbf{X}_{r}$ is an individual different from $\mathbf{X}_{t-1}$. Individual $\mathbf{X}_{r}$ can be obtained according to the following strategies: $\mathrm{Sg}$;

1. sampled from all individuals of the current generation:

2. sampled from the matrix $\mathbf{B}$ of individual past bests: $\mathrm{Pb}$;

3. sampled as a uniform recombination from the individuals of the current generating: $\mathrm{Sg}$-rnd;

4. sampled as a uniform recombination within the matrix B: Pb-rnd.

Note that the computation of the movement rule must take into account the difference between the fitness of $\mathbf{X}_{r}$ and of $\mathbf{X}_{t-1}$. If $\mathbf{X}_{r}$ is better than $\mathbf{X}_{t-1}$, then the individual must be attracted to $\mathbf{X}_{r}$ and, hence, the computation of the new velocity is according to (13); otherwise, the position of $\mathbf{X}_{r}$ and $\mathbf{X}_{t-1}$ in (13) must be swapped in order to repel the individual from $\mathbf{X}_{r}$, i.e. move away from a worst position. For the cases where uniform recombination is used, i.e. for strategies 3 and 4, the verification of the relative position of $\mathbf{X}_{r}$ and $\mathbf{X}_{t-1}$ in (13) must be done for every dimension of the optimization problem by comparing the fitness of the individuals selected to build $\mathbf{X}_{r}$ and the fitness of $\mathbf{X}_{t-1}$.

\section{Unit Commitment using DEEPSO}

DEEPSO is a metaheuristic developed for real valued spaces. Each individual represents of DEEPSO represents a possible UC solution. The dimension of the individuals is $N_{G}$ $\times N_{T}$, where $N_{G}$ is the number of units and $N_{T}$ is the number of operation periods. Given the mixed-integer nature of the UC problem, a simple rounding procedure was used: even though the search was carried out in a real valued space, the respective solution is rounded off to obtain an integer one.

Special functions are also used to guarantee that the UC constraints are enforced. These functions, which are labeled Function A and Function B, are applied after the rounding process and every time a new UC solution is obtained.

\section{1) Function $A$}

This function verifies whether constraints (3) and (4) are satisfied. In the case of the deterministic UC, the point forecast is used when (3) and (4) are verified whereas the average hourly wind power is used for the case of the stochastic UC.

If constraint (3) is violated, a non-committed unit is selected and its status changed to ON. The selection of the unit to be started up can be made randomly or by using a priority list. The priority to start up units is defined according to

$$
m s u_{k}=\frac{P C_{k}\left(\overline{P_{k}}\right)+S U C_{k}}{\overline{P_{k}} \times T U P_{k}} .
$$

The unit with the smallest $m s u_{k}$ is started up first. Only one of the two selection procedures is used to start up a unit and each of the procedures has a 50\% change of being selected. Units are started up until constraint (3) is verified. After this first procedure, constraint (4) is analyzed. If this constraint is not satisfied, units are removed from the schedule, either randomly or using a priority list. Accordingly, a unit is selected to be shut down if the following metric

$$
m s d_{k}=\frac{P_{k} \times T D N_{k}}{P C_{k}\left(P_{k}\right)} .
$$

is the smallest for all units with the ON status. Units are then progressively shut down, with a $50 \%$ probability of being selected randomly or by using the metric defined by (15), until constraint (4) is fulfilled. Function A is stopped only if constraints (3) and (4) are simultaneously fulfilled. If not, this function is rerun.

\section{2) Function $B$}

This function is used to repair the solutions that have generating units with minimum up and/or minimum down time constraints violated, i.e., it guarantees that (5) is enforced. Hence, this function starts by analyzing the schedule of each generating unit and check whether the minimum up and down times are verified. If they are not, the status of the unit is changed from OFF to ON for the minimum necessary periods of time that cause the violation of inequation (5).

\section{3) Greedy Improvement of the Global Best}

At the end of the every generation of DEEPSO, a simple algorithm is run to obtain a new $\mathbf{X}_{g b}$. The idea is to apply a small and greedy modification to $\mathbf{X}_{g b}$, by using the metric defined in (14), and generate a new UC solution. If the fitness of this new UC solution is better than the fitness of $\mathbf{X}_{g b}$, then the new global attractor is replaced. The idea is to speed up the search process and generate additional diversity to escape from local optima.

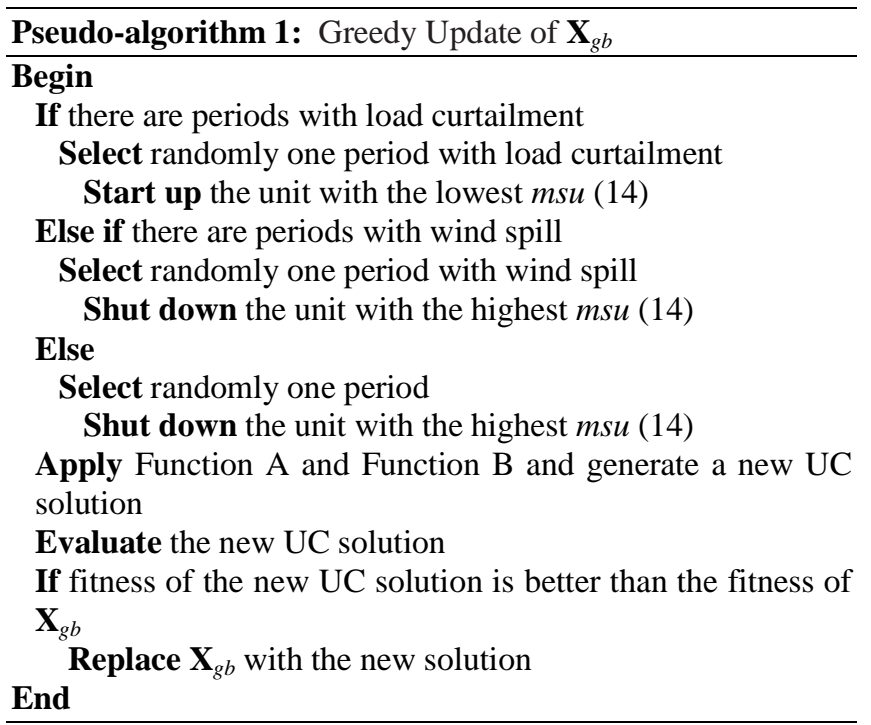


TABLE I. SYSTEM DATA

\begin{tabular}{|c|c|c|c|c|c|}
\hline Unit & $\begin{array}{c}\frac{P}{(\mathrm{MW})} \\
(\mathrm{MW})\end{array}$ & $\begin{array}{c}\bar{P} \\
(\mathrm{MW} / \mathrm{h})\end{array}$ & $\begin{array}{c}\text { Ramp Up } \\
(\mathrm{MW} / \mathrm{h})\end{array}$ & $\begin{array}{c}P_{0} \\
(\mathrm{MW})\end{array}$ \\
\hline 1 & 150 & 455 & 230 & 230 & 300 \\
\hline 2 & 150 & 455 & 230 & 230 & 300 \\
\hline 3 & 20 & 130 & 82.5 & 82.5 & - \\
\hline 4 & 20 & 130 & 82.5 & 82.5 & - \\
\hline 5 & 25 & 162 & 105 & 105 & - \\
\hline 6 & 20 & 80 & - & - & - \\
\hline 7 & 25 & 85 & - & - & - \\
\hline 8 & 10 & 55 & - & - & - \\
\hline 9 & 10 & 55 & - & - & - \\
\hline 10 & 10 & 55 & - & - & - \\
\hline
\end{tabular}

\section{4) Generation of the Initial Population}

The generation of the initial population follows a heuristic process. Firstly, the minimum wind power at each period is obtained from all wind power scenarios. After that, this value is subtracted from the hourly load to obtain a net load. Units are then started up from $t=1$ to $t=24$ to supply the net load plus the spinning reserve requirements using the priority list defined by the metric (14).

Function A and B are then applied to obtain a feasible UC solution. This solution is then artificially included in the population. The remaining individuals are obtained randomly. If these individuals represent infeasible solutions, Function A and $\mathrm{B}$ are applied.

\section{Scenario Clustering Technique}

The initial set of scenarios used in this paper refers to a day-ahead wind power forecasts in 2006, for a wind farm located in the state of Illinois. Time series of day-ahead deterministic point forecasts were obtained from the National Renewable Energy Laboratory's Eastern Wind Integration and Transmission Study [12]. To conceive wind power scenarios, wind power data (forecasted and realized) were used to train the uncertainty estimation model, as well as to generate scenarios of the forecasted wind power, according to the methodology introduced by Pinson et al. [5], which is equivalent to producing scenarios under a Monte Carlo process.

The scenario reduction method is based on a clustering technique [6]. It begins with the original large set of wind power scenarios; then, one finds, in the T-dimension space, the region with the highest probability density for the wind power. A cluster of scenarios in this region is defined and replaced by a best matching unit - its focal scenario, a representative element with a probability given as the probability of drawing any scenario within its cluster out of the whole sample set. This cluster is removed and the process is repeated until a stopping criterion is met.

This way, a set of clusters is defined, each associated to a focal element together with a probability value of its cluster. Here the $10 \%$ tolerance has been used during the scenario aggregation process.

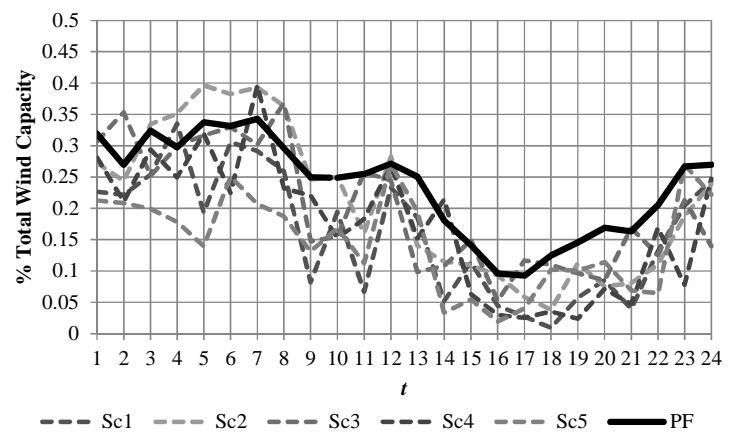

Figure 1. Illustration of the five most probable wind scenarios (Sc) and the point forecast (PF).

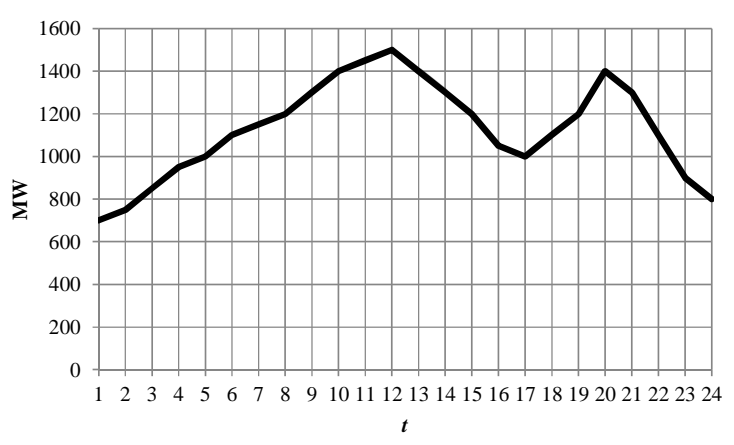

Figure 2. Hourly load.

\section{CASE STUDY}

A set of experiments were conducted to investigate the efficacy of the stochastic UC approach against the deterministic approach. For this purpose, a wind farm was added to the 10 generator system [10] and experiments were conducted for two different wind penetration scenarios: 250 MW and $750 \mathrm{MW}$ wind capacities. Wind power point forecasts for one operational day (24-hour) plus a set of 1000 equiprobable wind scenarios were also used. These scenarios were assigned to 406 clusters using the clustering technique previously described. Figure 1 shows the shape of the five most probable scenarios obtained with the clustering technique as well as the point forecast for that particular day.

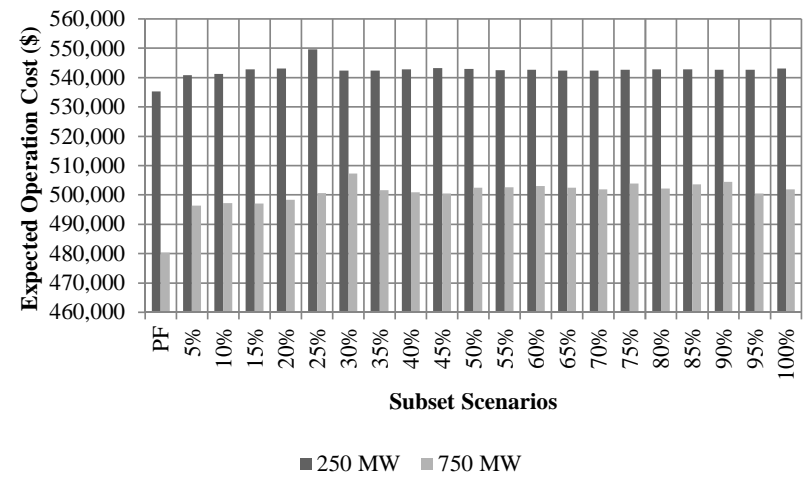

Figure 3. Expected operation cost for the $250 \mathrm{MW}$ and $750 \mathrm{MW}$ cases and for all UC solutions. 


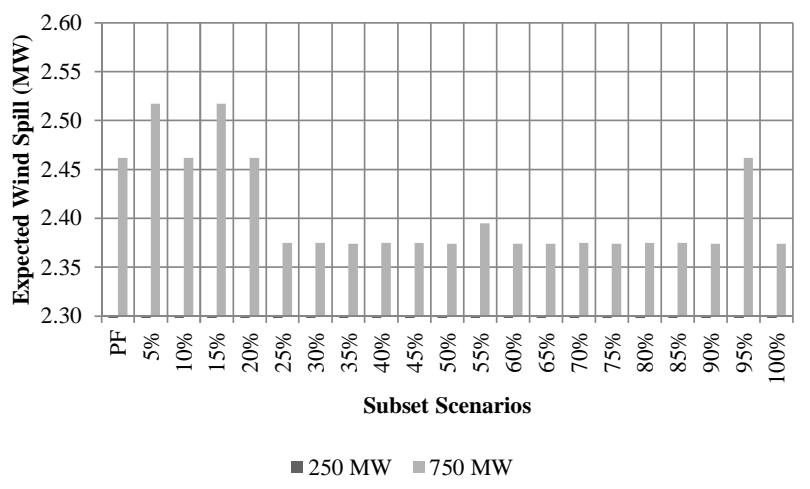

Figure 4. Expected wind spill for the $250 \mathrm{MW}$ and $750 \mathrm{MW}$ cases and for all UC solutions.

All relevant data of the 10 generator system can be found in [10]. As a simple illustration, Figure 2 shows the system hourly load. Table I contains additional data that is not available in [10], namely, the data used for the ramp rates of the generating units and the power production at the beginning of the commitment horizon $(t=0)$. It was also assumed that the spinning reserve is $10 \%$ of the hourly load. Constants M1 and $\mathrm{M} 2$ of (1) were set to $1 \times 10^{15}$ and $1 \times 10^{10}$, respectively, in order to spill wind before curtailing load. The DEEPSO strategy used was $\mathrm{Pb}$-rnd with mutation rate $\tau=0.6$, communication probability $P=0.7$, and a population of 20 individuals. DEEPSO was stopped after 500 generations.

Twenty-one UC solutions were obtained by DEEPSO. One of these UC solutions was obtained using the point forecast, i.e. by using the traditional deterministic approach. The remaining UC solutions were obtained using the stochastic approach by considering 20 different subsets of the 406 clusters of wind power scenarios. These subsets were obtained by ordering the clusters of scenarios by their underlying probability and selecting scenarios from the most probable one to the less probable until a given predefined number is reached. For instance, the subset that contains the lowest number of scenarios contains only the 5\% most probable scenarios from all 406, i.e. 20 scenarios, whereas the subset with the greatest number contains all 406 scenarios. The percentage of the scenarios included in the subset is used as its id.

The optimal dynamic economic dispatch considering wind spill and load curtailment for all 21 UC solutions was computed for all 406 wind scenarios to investigate the effect of using a stochastic UC approach instead of a deterministic approach on the expected operation cost (i.e. the startup cost plus the power production cost weighted by the corresponding scenario probability), on the expected wind power spill, and on the expected load curtailment.

The methodology was implemented in Java. All simulations were carried out in an Intel Core i7-2600@3.40 $\mathrm{GHz}$. The average time required to run the methodology proposed for the case of the point forecast was 64 minutes whereas the time required for all 406 scenarios was 186 minutes.
Figure 3 shows the expected operation cost for the 250 MW and 750 MW cases for all 21 UC solutions produced by DEEPSO, where PF stands for the UC solution obtained using the point forecast. First of all, this figure shows that the expected operation cost decreases as more wind power is added to system. This observation is consistent with the assumption that that wind power has zero cost: by integrating more wind, conventional generating units are replaced.

Secondly, Figure 3 reveals a steady increase of the expected operation cost as more wind scenarios are used by DEEPSO to generate UC solutions. This cost then stabilizes after a given subset of scenarios, exhibiting only slight variations as more scenarios are used. Beyond this threshold, which in the case of $250 \mathrm{MW}$ is $15 \%$ and $25 \%$ in the $750 \mathrm{MW}$ case, one can see that the expected operation cost does not increase nor decrease significantly by adding more wind scenarios to the generation of UC solutions process. The small differences observed can be explained by the inherent characteristics of metaheuristics-based optimization which do not guarantee that the global optimum has been reached.

As a matter of fact, metaheuristics can be trapped in local optima in some cases. The former statement is corroborated the bad performing UC solution obtained when using the $25 \%$ most probable scenarios for the $205 \mathrm{MW}$ case. To avoid this problem, DEEPSO needs to be run a considerable number of times for all cases and subset of scenarios such that several UC solutions are obtained for both the deterministic and stochastic approaches. These UC solutions would be analyzed individually and average values computed. However, this type of approach is cumbersome and very time consuming and is not necessary if the overall behavior or trend of the expected operation cost as more wind scenarios are used is to be compared.

Figure 4 depicts the average wind power spilled for the two wind power capacity cases and for all 21 UC solutions. In the $250 \mathrm{MW}$ case, there is no wind power spilled for all scenarios whereas there will always be wind spill for the 750 MW case. In the former case, the expected wind spilled gradually decreases as more wind scenarios are used by DEEPSO to build the UC solution. The expected wind spilled stabilizes after using $25 \%$ of the most probable scenarios, leading to the conclusion that that, for this case, there is no additional information gain by including more scenarios in the generation of UC solutions. Note that the computational effort increases as more scenarios are used by DEEPSO since the optimal dynamic economic dispatch for each scenario must be computed using Linear Programming for each new UC solution. Hence, considerable computational effort savings can be obtained if the number of scenarios is optimally estimated beforehand. Finally, there were some runs where DEEPSO has provided local optimum solutions, namely, in the cases of the subsets that contained $55 \%$ and $95 \%$ of the most probable scenarios.

Table II presents the expected load curtailment for the 21 UC solutions. The analysis of these results allows concluding for this particular day that the deterministic UC approach can be a risky option since it leads to the highest expected load curtailment. However, this value can be greatly reduced by 
using the stochastic approach with only $5 \%$ of the most probable wind scenarios: this approach resulted in a reduction on the expected load curtailment by more than 670 times in the case of $250 \mathrm{MW}$ and by more than 2320 for the case of $750 \mathrm{MW}$.

TABLE II. EXPECTED LOAD CURTAILMENT (MW)

\begin{tabular}{|c|c|c|c|c|c|}
\hline \multirow{3}{*}{$\begin{array}{c}\text { Subset } \\
(\%)\end{array}$} & \multicolumn{2}{|c|}{$\begin{array}{c}\text { Expected Load } \\
\text { Curtailment (MW) }\end{array}$} & \multirow{2}{*}{$\begin{array}{c}\text { Subset } \\
(\%)\end{array}$} & \multicolumn{2}{c|}{$\begin{array}{c}\text { Expected Load } \\
\text { Curtailment (MW) }\end{array}$} \\
\cline { 2 - 3 } & $\begin{array}{c}\text { Case } \\
250 \mathrm{MW}\end{array}$ & $\begin{array}{c}\text { Case } \\
750 \mathrm{MW}\end{array}$ & & $\begin{array}{c}\text { Case } \\
250 \mathrm{MW}\end{array}$ & $\begin{array}{c}\text { Case } \\
750 \mathrm{MW}\end{array}$ \\
\hline PF & 24.95 & 195.42 & 55 & - & - \\
\hline 5 & $3.72 \mathrm{E}-02$ & $8.42 \mathrm{E}-02$ & 60 & - & - \\
\hline 10 & $3.72 \mathrm{E}-02$ & $2.01 \mathrm{E}-02$ & 65 & - & - \\
\hline 15 & - & $2.32 \mathrm{E}-02$ & 70 & - & - \\
\hline 20 & - & - & 75 & - & - \\
\hline 25 & $2.80 \mathrm{E}-03$ & - & 80 & - & - \\
\hline 30 & - & - & 85 & - & - \\
\hline 35 & - & - & 90 & - & - \\
\hline 40 & - & - & 95 & - & - \\
\hline 45 & - & - & 100 & - & - \\
\hline 50 & - & - & & & \\
\hline & & & & & \\
\hline
\end{tabular}

Note that the expected load curtailment for the $250 \mathrm{MW}$ case is only zero after using the $30 \%$ most probable scenarios whereas $20 \%$ of scenarios is sufficient to eliminate the risk of load curtailment in the $750 \mathrm{MW}$ case. Note that, in the 250 MW case, there are UC solutions that were obtained using less than $30 \%$ of the most probable scenarios and still results in zero expected load curtailment: this indicates that the UC solution obtained for the $30 \%$ subset of scenarios is suboptimal.

While the former analysis was conducted for a single operational day, it can be concluded that the stochastic approach can greatly mitigate of the risk of high operational costs and adverse control measures such wind spill or load curtailment. Note that, in this case, it is possible to greatly reduce the risk of load curtailment only by using the $5 \%$ most probable scenarios (i.e. 20 scenarios out of the 406) in the generation of the UC schedule.

\section{CONCLUSIONS}

The presence of wind power in the generation portfolio of a system introduces a margin of uncertainty at least an order of magnitude larger than the uncertainty in load. Furthermore, ramp events in the wind speed are likely to cause considerable stress to the system, especially when it is based on slow reacting thermal units.

Many researchers agree that some form of stochastic optimization could be an answer to this concern. However, two problems remain: the large computational demand of stochastic programming when the number of scenarios is large and the selection of a representative small set of scenarios.

This paper presented a comparison between deterministic and stochastic approaches for Unit Commitment in a system with wind power generation, and provided results from experiments for a succession of a growing number of wind power scenarios. The relevance of the work presented may be summarized as follows: given that an adequate scenario reduction and clustering is performed, the stochastic programming problem may be solved with a reduced set of well-chosen equivalent scenarios, preserving the quality of the solution when compared to solving the problem for a very large set of scenarios.

The results of the tests presented clearly show that:

- a stochastic formulation provided UC solutions more robust than the ones from a deterministic solution based on a point forecast

- a well-chosen subset as small as of 5\% out of 1000 wind scenarios (generated by a Monte Carlo process from a wind-to-power model) may provide a high quality result by a considerable reduction of the expected load curtailment in the whole subset of scenarios

- the increase adoption of more scenarios does not add significant quality to the solutions.

Finally, the efficiency of the solving method is also a key factor in the practical feasibility of the method. The adoption of an efficient meta-heuristic such as DEEPSO allowed the tests to be fully performed although the paper is not proposing, as a result, that such should be the algorithmic tool to adopt in a professional environment.

\section{REFERENCES}

[1] L. Wu, M. Shahidehpour, and T. Li, "Cost of reliability analysis based on stochastic unit commitment," IEEE Trans. Power Syst., vol. 23, no. 3, pp. 1364-1374, 2008.

[2] C. Zhao and Y. Guan, "Unified stochastic and robust unit commitment," IEEE Trans. Power Syst., vol. 28, no. 3, pp. 3353-3361, 2013.

[3] P. Xiong and P. Jirutitijaroen, "A stochastic optimization formulation of unit commitment with reliability constraints," IEEE Trans. Smart Grid, vol. 4, no. 4, pp. 2200-2208, 2013.

[4] C. Zhao, Q. Wang, J. Wang and Y. Guan, "Expected Value and Chance Constrained Stochastic Unit Commitment Ensuring Wind Power Utilization," IEEE T. Power S., vol. 29, no. 6, pp. 2696-2705, May 2014

[5] P. Pinson. H. Madsen. H. A. Nielsen. Papaefthymiou and B. Klöckl, "From Probabilistic Forecasts to Statistical Scenarios of Short-term Wind Power Production," Wind Energy, v. 12, no. 1, pp. 51-62, Jan. 2009

[6] J. Sumaili, H. Keko, V. Miranda, A. Botterud, J. Wang, "ClusteringBased Wind Power Scenario Reduction Technique," in Proc. $201117^{\text {th }}$ Power Systems Computation Conference (PSCC), vol. 1, pp. 391-397.

[7] V. Miranda and R. Alves, "Differential Evolutionary Particle Swarm Optimization (DEEPSO): a successful hybrid," $11^{\text {th }}$ Brazilian C. Comput.1 Intelligence (BRICS-CCI), Porto de Galinhas, Brazil, 2013.

[8] Mokhtar S. Bazaraa, John J. Jarvis, and Hanif D. Sherali, Linear Programming and Network Flows, 4th ed., New Jersey:John Wiley \& Sons, Inc., 2009.

[9] Gurobi Optimization. [Online] Available: http://www.gurobi.com/

[10] S. A. Kazarlis, A. G. Bakirtzis, and V. Petridis, "A genetic algorithm solution to the unit commitment problem," IEEE Trans. Power Syst., vol. 11, no. 1, pp. 83-92, Feb. 1996.

[11] A. J. Wood, B. F. Wollenberg, and G. B. Sheblé, Power Generation Operation and Control, 3rd ed., New Jersey:John Wiley \& Sons, Inc., 2014.

[12] (X1) Eastern Wind Integration and Transmission Study (EWITS), National Renewable Energy Laboratory (NREL) http://www.nrel.gov/wind 